\section{Evolutionary theory}

\section{Hamilton's rule OK}

\section{From Alan Grafen}

THE darwinian principal of natural selection was extended by Hamilton in 1964 to the evolution of social behaviour'. This extension is central to much of today's evolutionary biology, but it is something of a sport among mathematical biologists to cast doubt on its validity. Now David Queller, writing on page 366 of this issue ${ }^{2}$, presents a simple and powerful derivation of Hamilton's result that should go some way towards convincing the sceptics.

Hamilton's extension to darwinism is enchantingly simple and has come to be known as 'Hamilton's rule'. In an uncomplicated world, the morphology and behaviour of an individual would affect the number of offspring it had in its lifetime but not the number of offspring other individuals had. Given that individuals do interact, though, the question is how will selection cause animals to value each other's reproduction? Will they place no value on it - that is, will selection favour forms according only to their own number of offspring'? Or will they place equal value on their own reproduction and that of others - that is, will selection favour those forms that produce more offspring in total for the species? Hamilton's answer was intermediate between these two ex-

\section{Long-running experiments (I)}

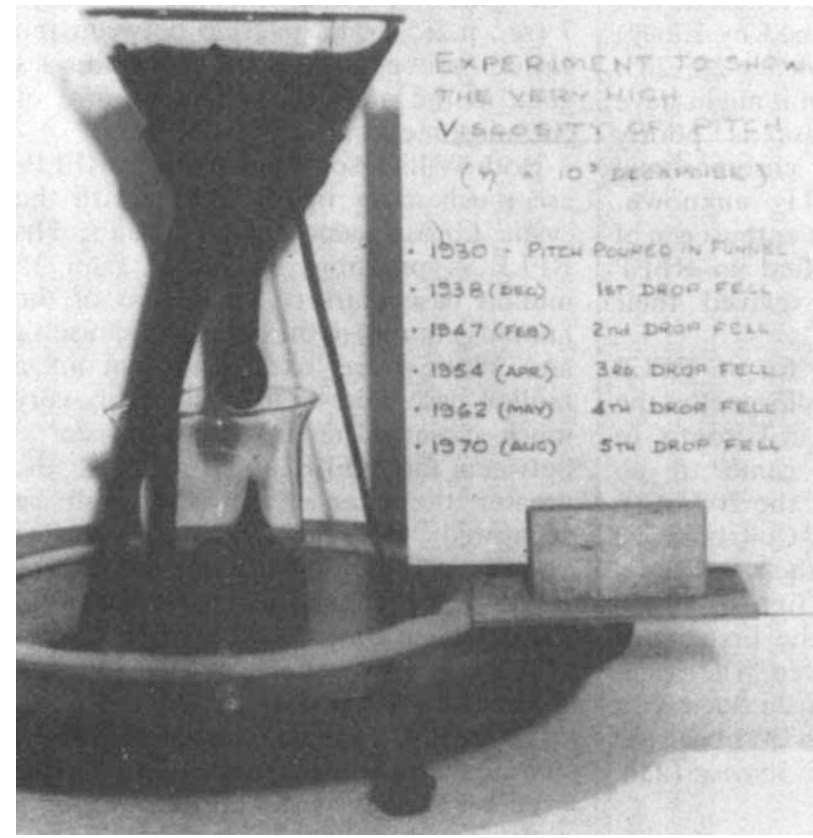

Only six drops have fallen, the latest in April 1979, since this experiment was set up in 1930. Its aim is to demonstrate and measure the fluidity and very high viscosity of pitch. The experiment, which continues in the Department of Physics at the University of Queensland, Australia, is one of three longstanding experiments considered in detail in the European Journal of Physics 5, 193-200 (1984), along with a request for accounts of further examples. tremes. He showed mathematically that two individuals were related. Identical would value each other's offspring ters would value each other's offspring half as much as offspring of their own; and on. The evolution of a social trait then on an 'inclusive fitness' calculation, in which all the effects of the trait on the are added together, weightexpense of others. nswer this question. Queller's contribution is to provide a very simple and direct proof of Hamilton's rulc, as an antidote to I feel is the biologically unsympatheusually applied. The positive feature of Queller's approach is that it sacrifices an element of mathematical rigour for the sake of deriving a much more general result, and deriving it with clarity and simplicity.

Complete recursion, or dynamic sufficiency, is the element of rigour sacrificed by Queller's approach. In a simple model (one locus, two alleles) in a diploid species, we can say that a rigorous model uses the frequencies of all three genotypes in one generation to predict all three genotype frequencies in the next. Complete recursion is the use of those genotype frequencies for predicting the genotype frequencies in the following generation. and so on. Queller's method has an incomplete recursion. because it uses all three genotype frequencies in one generation to predict the frequencies of the two genes in the next. Without making some assumption (usually that the Hardy-Weinberg equilibrium holds). genotype frequencies cannot be calculated from gene frequencies, and so it is impossible to apply Queller's equations repeatedly to predict gene frequencies in succeeding generations.

That is the cost of Queller's simplification. Whether it is serious depends on one's point of view. One view is that incomplete recursions are unsafe, because to predict gene frequencies into the future they must make some false assumption (such as Hardy - Weinberg), and since the consequences of the assumption may not be innocuous, the only way to provide a secure argument is not to make the assumption in the first place. But not all conclusions that we draw from evolutionary models depend on predicting gene frequencies in the future. Queller's model predicts whether a character increases or decreases from one generation to the next, and shows that it depends on a form of Hamilton's rule. He makes no claim about dynamic sufficiency (although he does unguardedly claim his model is "exact", a term usually reserved for completely recursive models). nor is any needed for his conclusion to be both interesting and important.

The advantages gained by forgoing complete recursion are great. Since Queller's model does not specify what causes the genetic similarity between donor and recipient, his result holds whether the cause is a recent common ancestry, heterogeneity of the population, or the tendency of individuals to interact preferentially with like genotypes for other reasons. His interactants need not comprise mutually exclusive and exhaustive groups. Completely recursive models are hard work: they assume (contingently, not necessarily) mutually exclusive and exhaustive groups, and they must specify exactly how genetic similarity is caused. For example. a model in which each group comprises half siblings would be quite different from one in which each group was formed by picking individuals at random from two sets of full siblings. Queller's approach shows that the relatedness is one quarter in each case. and so the two are immediately scen to be essentially similar. Completely recursive models would be very different and - though for no very clear reason - would eventually give the same answers as each other (and as Qucller's model) in the matters important for the evolution of a character. The models would differ in details of interest to the population geneticist; for instance. they would have different kinds of polymorphic equilibria when the two alleles were so similar in effect as to be empirically indistinguishable.

While. in my view. Queller's trade-off leaves him as a champion of Hamilton's rule in one battle. he has been too ready to concede defeat on its behalf in another. Hamilton's rule was first derived for additive social interactions, in which the 